\title{
Using selective chromogenic plates to optimize isolation of group B Streptococcus in pregnant women
}

\author{
Romano Mattei', Roberta Creti' ${ }^{2}$, Maria Nardone' \\ I Laboratorio Analisi Chimico-Cliniche e Microbiologiche, Ospedale Campo di Marte, Lucca. \\ 2 Reparto Malattie Batteriche Respiratorie e Sistemiche, Dipartimento di Malattie Infettive, Parassitarie ed Immunomediate, \\ Istituto Superiore di Sanità, Roma
}

Key words: Group B Streptococcus, Streptococcus agalactiae, GBS, Pregnant women, PAI Utilizzo di piastre cromogeniche selettive per ottimizzare l'isolamento di Streptococco gruppo B
in gravidanza

\section{SUMMARY}

Group B Streptococcus (GBS) remains the leading cause of severe bacterial infections (sepsis, meningitis, pneumonia) in neonates.

We compared the detection of GBS from recto-vaginal swabs on blood agar and two chromogenic media and evaluated their antibiotic susceptibility. A total of I35I swabs were taken from pregnant women at 35-37 weeks of gestation. Following enrichment in Todd Hewitt broth + nalidixic acid and colistin, the samples were plated on Columbia CNA agar (CNA), chromID Strepto B agar (STRB) and Granada Agar (GRAN), respectively. GBS were found in $22.4 \%$ of recto-vaginal swabs from pregnant women. Sensitivity, specificity, positive and negative predictive values of GBS detection were $88 \%, 88 \%, 81 \%$ and $96 \%$ for CNA, $99 \%, 97 \%, 90 \%$ and $99 \%$ for STRB and $94 \%, 99 \%, 98 \%$ e $99 \%$ for GRAN; Cohen's $k$ index concordances for CNA, STREB and GRAN were $0.68,0.92$ and 0.96 , respectively. All isolates were susceptible to penicillin, whereas resistances of erythromycin and clindamycin were $40 \%$ and $42 \%$, respectively.

To conclude, selective broth enrichment combined with chromogenic plates is recommended for GBS screening in pregnant women.

\section{INTRODUZIONE}

Lo streptococco gruppo B (GBS) o Streptococcus agalactiae è emerso negli anni '70 come la principale causa infettiva di morbilità e mortalità neonatale negli Stati Uniti $(9,12)$. La malattia si manifesta con una forma precoce (entro sette giorni dalla nascita, ma soprattutto entro le prime 24 ore di vita) come sepsi, polmonite, distress respiratorio e in misura minore meningite e una forma tardiva (fino a tre mesi di vita) soprattutto come meningite, spesso con esiti permanenti.

La colonizzazione con GBS nel tratto genito-urinario o gastrointestinale è il principale fattore di rischio per la malattia. Studi clinici hanno dimostrato che la somministrazione di antibiotici per via endovenosa durante il travaglio alle donne a rischio per la trasmissione di GBS ai loro neonati può prevenire la malattia invasiva ad esordio precoce $(3,10)$. La profilassi antibiotica intrapartum (PAI) viene attuata tramite somministrazione di penicillina G; l'uso di ampicillina è considerata una valida alternativa. Alle partorienti candidate alla PAI ma allergiche alla penicillina viene somministrata cefazolina se considerate a basso rischio di anafilassi (assenza di anafilassi, angioedema, distress respiratorio o orticaria in seguito ad una precedente somministrazione di ampicillina) o clindamicina se ad alto rischio di anafilassi. Se il ceppo GBS, isolato nello screening prenatale, risulta resistente a eritromicina e clindamicina dovrebbe essere somministrata la vancomicina (5). Il periodo compreso fra le ultime cinque settimane ed il parto è quello raccomandato dal CDC per la ricerca dello stato di colonizzazione in corso di gravidanza; il prelievo eseguito da retto e vagina è consigliato perché più sensibile rispetto al prelievo esclusivamente vaginale (5-8). Il mezzo convenzionale per la coltura di GBS è l'arricchimento in brodo selettivo seguito dalla subcoltura su piastre di agar sangue, ma sono disponibili anche agar cromogeni che subiscono cambiamento di colore in presenza di colonie di $\operatorname{GBS}(2,7$, $13,14,16)$.

Lo scopo del nostro lavoro è stato quello di valutare la migliore capacità diagnostica per la ricerca di GBS su tampone vagino-rettale nelle donne in stato di gravidanza tra piastre cromogeniche e coltura convenzionale su agar Columbia CNA dopo

\section{Corresponding author: Romano Mattei}

Laboratorio Analisi Chimico Cliniche e Microbiologiche, Ospedale Campo di Marte, 55 100 Lucca

Tel: 3487424032; Fax 0583970402

E-mail: r.mattei@usl2.toscana.it 
arricchimento in brodo selettivo.

\section{MATERIALI E METODI}

Nel periodo luglio 2011 - marzo 2012 sono stati raccolti 1353 tamponi vagino-rettali da donne in stato di gravidanza a 35 - 37 settimane di gestazione.

Il prelievo è stato effettuato da ostetriche facendo ruotare il tampone contro la parete vaginale alla porzione mediana della volta; successivamente lo stesso tampone è stato inserito $1.5-2 \mathrm{~cm}$ oltre lo sfintere anale per toccare le cripte anali con delicato movimento rotatorio.

I tamponi inseriti in terreno di trasporto Amies sono stati inviati entro 2 ore al Laboratorio Analisi dell'Ospedale di Lucca. I tamponi sono stati inoculati nel brodo di arricchimento Todd Hewitt Broth reso selettivo dall'aggiunta di gentamicina e acido nalidixico (Becton Dickinson) e quindi, dopo $18-24$ ore di incubazione a $37^{\circ} \mathrm{C}$, venivano subcoltivati in tre terreni selettivi: Columbia agar con $5 \%$ di sangue di montone addizionato con 10 $\mathrm{mg} / \mathrm{ml}$ di colistina e $15 \mathrm{mg} / \mathrm{ml}$ di acido nalidixico (CNA, Becton Dickinson), Granada ${ }^{\mathrm{TM}}$ agar (GRAN, bioMérieux) e chromID ${ }^{\text {TM }}$ Strepto B agar (STREB, bioMérieux, Marcy l'Etoile, France).

Le colonie esaminate e sottoposte a successiva identificazione ed eventuale antibiogramma sono state: le colonie beta-emolitiche su CNA, le colonie con pigmento giallo-arancio su GRAN, le colonie da rosa pallido a rosse su STREB. L'identificazione di GBS veniva raggiunta con test di agglutinazione per la definizione del poli- saccaride di gruppo e con prove biochimiche eseguite su VITEK 2 (bioMérieux).

Su VITEK 2 veniva eseguito anche l'antibiogramma, utilizzando i criteri di interpretazione dell'European Committee on Antimicrobial Susceptibility Testing (www.eucast.org) e sui ceppi eritromicina-resistenti e clindamicina-sensibili veniva eseguito il D-test per stabilire la resistenza inducibile alla clindamicina.

Per valutare il grado di concordanza dei tre terreni di coltura rispetto al numero di donne GBS positive è stato utilizzato il coefficiente statistico kappa di Cohen.

\section{RISULTATI}

Dei 1353 tamponi vagino-rettali analizzati per la ricerca di Streptococcus agalactiae, 301 sono risultati positivi $(22.4 \%)$. Il numero totale di soggetti positivi è stato preso come $100 \%$, per calcolare sensibilità, specificità e valore predittivo negativo e positivo dei tre diversi terreni di coltura (Tabella 1). I falsi negativi su agar CNA e GRAN sono ascrivibili a ceppi di GBS non emolitici. I falsi positivi sono stati 130 su agar CNA, per lo più dovuti a ceppi di Enterococcus faecalis beta-emolitici, 33 su agar STREB, nella maggior parte dei casi da ceppi di Streptococcus anginosus e da alcuni ceppi di Streptococcus constellatus, Lactococcus species ed Enterococcus faecalis; infine le colture false positive su agar GRAN sono risultate 5, causate da ceppi di Enterococcus faecalis e Lactococcus species.

Tabella I. Sensibilità, specificità, valori predittivi positivi e negativi per i tre metodi di coltura sui I353 campioni esaminati di cui 301 positivi

\begin{tabular}{ccccc}
\hline $\begin{array}{c}\text { Terreno } \\
\text { di Coltura }\end{array}$ & Sensibilità $\%$ & Specificità \% & $\begin{array}{c}\text { Valore predittivo } \\
\text { positivo \% }\end{array}$ & $\begin{array}{c}\text { Valore predittivo } \\
\text { negativo \% }\end{array}$ \\
\hline CNA & 88 & 88 & 81 & 96 \\
\hline STREB & 99 & 97 & 90 & 99 \\
\hline GRAN & 94 & 99 & 98 & 99 \\
\hline
\end{tabular}

Il grado di concordanza dei tre terreni di coltura rispetto al numero di donne GBS positive utilizzando il coefficiente statistico di Cohen è rappresentato in tabella 2 .

Tabella 2. Interpretazione del coefficiente statistico $k$ di Cohen e risultati per i vari terreni di coltura

\begin{tabular}{ccc}
\hline Valori del coefficiente K di Cohen & Interpretazione & Terreno di coltura \\
\hline $0-0.4$ & Scarsa concordanza & \\
\hline $0.4-0.6$ & Discreta concordanza & CNA $(\mathrm{k}=0.68)$ \\
\hline $0.6-0.8$ & Buona concordanza & STREB $(\mathrm{k}=0.92), \mathrm{GRAN}(0.96)$ \\
\hline $0.8-1$ & Ottima concordanza &
\end{tabular}

I risultati dei test di sensibilità sono mostrati in tabella 3. 
Tabella 3. Percentuali di resistenza agli antibiotici dei 30 I ceppi di GBS isolati

\begin{tabular}{cc}
\hline & $\%$ isolati resistenti \\
\hline Antibiotico & $0 \%$ \\
\hline Penicillina & $0 \%$ \\
\hline Vancomicina & $0 \%$ \\
\hline Teicoplanina & $0 \%$ \\
\hline Linezolid & $0 \%$ \\
\hline Tigeciclina & $40 \%$ \\
\hline Clindamicina* & $42 \%$ \\
\hline Eritromicina & $6 \%$ \\
\hline Levofloxacina & $0.3 \%$ \\
\hline Moxifloxacina & $91 \%$ \\
\hline Tetraciclina & $3 \%$ \\
\hline Trimethoprim/sulfametoxazolo & \\
\hline
\end{tabular}

\section{DISCUSSIONE}

Lo screening microbiologico eseguito sulle donne in stato di gravidanza a 35 - 37 settimane di gestazione ha evidenziato una prevalenza delle colonizzazioni da Streptococcus agalactiae pari al $22.4 \%$, simile ad altre indagini epidemiologiche condotte nel nostro Paese, utilizzando la procedura CDC di arricchimento in brodo $(1,4,6,15)$.

I terreni cromogenici presentano sensibilità e specificità superiori al terreno al sangue; lo studio mostra una sottostima dei casi di colonizzazione utilizzando esclusivamente il terreno al sangue, in accordo con altri lavori $(2,7,8,13,14,16)$.

Tutti gli isolati di GBS sono risultati sensibili alla penicillina e vancomicina, mentre sono risultati resistenti a clindamicina ed eritromicina, agenti alternativi per la profilassi intraparto in donne con alto rischio di anafilassi, rispettivamente il 40 e $42 \%$ dei ceppi di GBS isolati.

In conclusione, i nostri risultati indicano la necessità di utilizzare terreni cromogenici per l'isolamento di GBS e la necessità di eseguire sui ceppi isolati l'antibiogramma almeno in pazienti allergici alla penicillina. Il laboratorio, vista l'elevata frequenza d'isolamento di GBS con resistenza inducibile alla clindamicina, deve prevedere l'utilizzo di test di laboratorio validati per determinare tale tipo di resistenza.

\section{BIBLIOGRAFIA}

1. Berardi A, Lugli L, Baronciani D, et al. GBS Prevention Working Group of Emilia-Romagna. Group B Streptococcus early-onset disease in EmiliaRomagna: review after introduction of a screeningbased approach. Pediatr Infect Dis J 2010; 29: 115-21.

2. Bou G, Figueira M, Canle D, Cartelle M, Eiros JM, Villanueva R. Evaluation of group B streptococcus Differential Agar for detection and isolation of Streptococcus agalactiae. Clin Microbiol Infect 2005; 11: 676-8.

3. Boyer KM, Gotoff SP. Prevention of early-onset neonatal group B streptococcal disease with selective intrapartum chemoprophylaxis. N Engl J Med 1986;
314: 1665-9.

4. Busetti M, D'Agaro P, Campello C. Group B Streptococcus prevalence in pregnant women from North-Eastern Italy: advantages of a screening strategy based on direct plating plus broth enrichment. $J$ Clin Pathol 2007; 60: 1140-3.

5. CDC. Prevention of perinatal group B streptococcal disease: revised guidelines from CDC. MMWR 2010; 51 (No. RR-10).

6. Creti R. Infezioni neonatali precoci e tardive da streptococco di gruppo B in Italia. 2011. Rapporti Istisan 11/7, 69 pp.

7. De La Rosa M, Villareal R, Vega D, Miranda C, Martinezbrocal A. Granada medium for detection and identification of group B streptococci. J Clin Microbiol 1983; 18: 779-85.

8. El Aila NA, Tency I, Claeys G, et al. Comparison of different sampling techniques and of different culture methods for detection of group B streptococcus carriage in pregnant women. BMC Infect Dis. 2010; 10: 285.

9. Franciosi RA, Knostman JD, Zimmerman RA. Group B streptococcal neonatal and infant infections. $J$ Pediatr 1973; 82: 707-18.

10. Lim DV, Morales WJ, Walsh AF, Kazanis D. Reduction of morbidity and mortality rates for neonatal group B streptococcal disease through early diagnosis and chemoprophylaxis. J Clin Microbiol 1986; 23: 489-92.

11. Kovavisarach E, Sa-adying W, Kanjanahareutai S. Comparison of combined vaginal-anorectal, vaginal and anorectal cultures in detecting of group B streptococci in pregnant women in labor. Journal of the Medical Association of Thailand [Chotmaihet thangphaet] 2007; 90: 1710-4.

12. McCracken GH, Jr. Group B streptococci: the new challenge in neonatal infections. J Pediatr 1973; 82: 703-6.

13. Poisson DM, Chandemerle M, Guinard J, Evrard ML, Naydenova D, Mesnard L. Evaluation of CHROMagar StrepB: A new chromogenic agar medium for aerobic detection of group B streptococci in perinatal samples. J Microbiol methods 2010; 82: 238-42.

14. Regnath T, Ignatius R. High stability of a new Granada medium agar that allows rapid and accurate detection of colonization with group B streptococci in pregnant women. Eur J Clin Microbiol Infect Dis 2009; 28: 1487-9.

15. Savoia D, Gottimer C, Crocilla C, Zucca M. Streptococcus agalactiae in pregnant women: phenotypic and genotypic characters. $J$ Infect 2008; 56: 120-5. 
16. Tazi A, Reglier-Poupet H, Dautezac F, Raymond J, Poyart C. Comparative evaluation of Strepto B ID chromogenic medium and Granada media for the detection of group B Streptococcus from vaginal samples of pregnant women. J Microbiol Methods 2008;
73: $263-5$.

17. Van Dyke MK, Phares CR, Lynfield R, et al. Evaluation of universal antenatal screening for group B Streptococcus. N Engl J Med 2009; 360: 2626-36. 